\title{
Symmetric Spaces and Fixed Points of Generalized Contractions
}

\author{
Sarah Alshehri, ${ }^{1}$ Ivan Aranđelović, ${ }^{2}$ and Naseer Shahzad ${ }^{3}$ \\ ${ }^{1}$ Department of Mathematics, Science Faculty for Girls, King Abdulaziz University, P.O. Box 4087, Jeddah 21491, Saudi Arabia \\ ${ }^{2}$ Faculty of Mechanical Engineering, University of Belgrade, Kraljice Marije 16, 11000 Beograd, Serbia \\ ${ }^{3}$ Department of Mathematics, King Abdulaziz University, P.O. Box 80203, Jeddah 21859, Saudi Arabia
}

Correspondence should be addressed to Naseer Shahzad; nshahzad@kau.edu.sa

Received 28 September 2013; Accepted 30 December 2013; Published 5 March 2014

Academic Editor: E. Karapinar

Copyright (C) 2014 Sarah Alshehri et al. This is an open access article distributed under the Creative Commons Attribution License, which permits unrestricted use, distribution, and reproduction in any medium, provided the original work is properly cited.

Some fixed point results in semi-metric spaces as well as in symmetric spaces are proved. Applications of our results to probabilistic spaces are also presented.

\section{Introduction}

There have been a number of generalizations of metric space. Two of them are the notions of symmetric spaces and semimetric spaces introduced and studied by Wilson [1]. For historical remarks about these spaces see [2]. Fixed point theory of various classes of maps in a metric space and its generalizations has been studied by a number of authors; see, for example, [3-9] and the references cited therein. In 1976, Cicchese proved the first fixed point theorem for contractions in semi-metric spaces. Further fixed point results for this class of spaces were obtained by Jachymski et al. [10], Hicks and Rhoades [11], Aamri and El Moutawakil [12], Aamri et al. [13], Zhu et al. [14], Miheţ [15], Imdad et al. [16], Aliouche [17], and Radenović and Kadelburg [18]. For more information on fixed point theory in symmetric spaces and semi-metric spaces, we refer the reader to [2].

In this paper we prove some fixed point results in semi-metric spaces and symmetric spaces. We also present applications of our results to probabilistic spaces. Our results generalize earlier results obtained by Aranđelović and Kečkić [2], Browder [19], Walter [20], and Maiti et al. [21].

\section{Preliminary Notes}

A symmetric space is a pair $(X, d)$ consisting of a nonempty set $X$ and a function $d: X \times X \rightarrow[0, \infty)$ such that for all $x, y$ in $X$ the following conditions hold:
(W1) $d(x, y)=0$ if and only if $x=y$;

(W2) $d(x, y)=d(y, x)$.

Let $(X, d)$ be symmetric space. The open ball with center $x \in X$ and radius $r>0$ is defined by

$$
B(x, r)=\{y \in X: d(x, y)<r\} .
$$

Also if $A$ is a subset of $X$, then

$$
\operatorname{diam}(A)=\sup \{d(x, y): x, y \in A\}
$$

denotes the diameter of $A$.

Many properties and notions in symmetric spaces are similar to those in metric spaces (but not all, because of the absence of the triangle inequality). For example, a sequence $\left\{x_{n}\right\} \subseteq X$ is said to be $d$-Cauchy sequence if given $\varepsilon>0$ there is $N \in \mathbb{N}$ such that $d\left(x_{m}, x_{n}\right)<\varepsilon$, for all $m, n \geqslant N$.

In every symmetric space $(X, d)$ one may introduce the topology $\tau_{d}$ by defining the family of closed sets as follows: a set $A \subseteq X$ is closed if and only if for each $x \in X, d(x, A)=0$ implies $x \in A$, where

$$
d(x, A)=\inf \{d(x, a): a \in A\} .
$$

The following conditions can be used as partial replacements for the triangle inequality's absence in the symmetric space $(X, d)$ :

$$
\begin{aligned}
& \text { (W3) } \lim _{n \rightarrow \infty} d\left(x_{n}, x\right)=0 \text { and } \lim _{n \rightarrow \infty} d\left(x_{n}, y\right)=0 \text { imply } \\
& x=y
\end{aligned}
$$


(W4) $\lim _{n \rightarrow \infty} d\left(x_{n}, x\right)=0$ and $\lim _{n \rightarrow \infty} d\left(x_{n}, y_{n}\right)=0$ imply $\lim _{n \rightarrow \infty} d\left(y_{n}, x\right)=0$;

(W) $\lim _{n \rightarrow \infty} d\left(x_{n}, y_{n}\right)=0$ and $\lim _{n \rightarrow \infty} d\left(y_{n}, z_{n}\right)=0$ imply $\lim _{n \rightarrow \infty} d\left(x_{n}, z_{n}\right)=0$;

(JMS) $\lim _{n \rightarrow \infty} d\left(x_{n}, y_{n}\right)=0$ and $\lim _{n \rightarrow \infty} d\left(y_{n}, z_{n}\right)=0$ imply $\lim _{n \rightarrow \infty} d\left(x_{n}, z_{n}\right) \neq \infty$;

(CC) $\lim _{n \rightarrow \infty} d\left(x_{n}, x\right)=0$ implies $\lim _{n \rightarrow \infty} d\left(x_{n}, y\right)=$ $d(x, y)$;

(SC) $\lim _{n \rightarrow \infty} d\left(x_{n}, x\right)=0$ implies $\varlimsup_{n \rightarrow \infty} d\left(x_{n}, y\right) \leq$ $d(x, y)$;

(MT) there exists $s \geqslant 1$ such that for any $x, y, z \in X$

$$
d(x, z) \leqslant s(d(x, y)+d(y, z)) .
$$

The properties (W3) and (W4) were induced by Wilson [1], (W) by Miheţ [15], (JMS) by Jachymski et al. [10], (CC) by Cho et al. [22] and earlier by Borges [23] (as 1-continuity property), (MT) by Czerwik [24] (see also [25]), and (SC) by Aranđelović and Kečkić [2].

Next statement gives the characterization of symmetric space which satisfies the property (JMS).

Proposition 1 (Jachymski et al. [10]). Let $(X, d)$ be a symmetric space. Then the following conditions are equivalent.

(i) $(X, d)$ satisfies property (JMS).

(ii) There exists $\delta, \eta>0$ such that for any $x, y, z \in X$,

$$
d(x, z)+d(z, y)<\delta \text { implies that } d(x, y)<\eta \text {. }
$$

(iii) There exists $r>0$ such that

$$
\sup \{\operatorname{diam}(B(x, r)): x \in X\}<\infty .
$$

The convergence of a sequence $\left\{x_{n}\right\}$ in the topology $\tau_{d}$ need not imply $d\left(x_{n}, x\right) \rightarrow 0$, although the converse is true (see Proposition 2).

The following two propositions have been well known for a long time, but for the convenience of the reader we will state them without proofs, which can also be found in [2].

Proposition 2. If $(X, d)$ is a symmetric space, then the family $\{B(x, r): r>0\}$ forms a local basis at $x$. Also, if $d\left(x_{n}, x\right) \rightarrow 0$, then $x_{n} \rightarrow x\left(\right.$ or $\left.\lim _{n \rightarrow \infty} x_{n}=x\right)$ in the topology $\tau_{d}$.

Definition 3. A topological space $(X, \tau)$ is semimetrizable if there is a symmetric function $d: X \times X \rightarrow \mathbf{R}$ such that $\tau_{d}=\tau$ and that the mapping

$$
X \supseteq A \longmapsto c(A)=\{x \in X: d(x, A)=0\}
$$

is the closure operator in $\tau_{d}$. In terms of $d$ it can be expressed by saying that the operator $c$ is idempotent. In this case we say that $(X, d)$ is semi-metric space; $d$ is said to be semi-metric function on $X$ (or admissible semi-metric for $(X, \tau)$ ).
It is worth mentioning that this basis need not consist of open sets. Moreover, in [26], a semimetrizable space $(X, \tau)$ was constructed with the property that, for any $d$ that generates $\tau$, there exist $x \in X$ and $r>0$ such that $B(x, r)$ is not open.

Proposition 4. Let $(X, d)$ be a symmetric space. Then $(X, d)$ is a semi-metric space if and only if the following conditions hold.

(1) $\left(X, \tau_{d}\right)$ is first countable.

(2) For any sequence $\left\{x_{n}\right\} \subseteq X, d\left(x_{n}, x\right) \rightarrow 0$ is equivalent to $x_{n} \rightarrow x\left(\right.$ or $\left.\lim _{n \rightarrow \infty} x_{n}=x\right)$ in the topology $\tau_{d}$.

Example 5. Let $X=\mathbb{N}$. Define $d: X \times X \rightarrow[0, \infty)$ by

$$
d(x, y)= \begin{cases}2^{1 / 2^{\min \{x, y\}}}-1 & \text { if }|x-y|=1 \\ 3 & \text { if }|x-y| \geqslant 2 \\ 0 & \text { if } x=y\end{cases}
$$

Then $(X, d)$ is a $d$-Cauchy complete semi-metric.

A symmetric space $(X, d)$ is said to be $d$-Cauchy complete if every $d$-Cauchy sequence converges to some $x \in X$ in the topology $\tau_{d}$, and it is said to be $d$-weakly complete if every decreasing sequence $\left\{F_{n}\right\}$ of nonempty closed subsets, such that there exists a sequence $\left\{x_{n}\right\}, x_{n} \in F_{n}$ with $F_{n} \subseteq B\left(x_{n}, 2^{-n}\right)$ has a nonempty intersection.

Next statement was proved in [27] (see also [28]).

Proposition 6 (Galvin and Shore [27]). Let $(X, d)$ be a semimetric space. Then the following are equivalent:

(1) $\left(X, \tau_{d}\right)$ is d-weakly complete;

(2) every d-Cauchy sequence in $X$ has a convergent subsequence;

(3) every decreasing sequence $\left\{F_{n}\right\}$ of nonempty closed subsets of $X$ such that $\operatorname{diam}\left(F_{n}\right) \leqslant 2^{-n}$ for each $n$ has a nonempty intersection.

Let $X$ be a nonempty set and $f: X \rightarrow X$. Then $z \in X$ is called a fixed point of $f$ if $z=f(z)$. Let $x \in X$. The sequence $\left\{x_{n}\right\}$ defined by $x_{n}=f^{n}(x)$ is called the sequence of Picard iterates of $f$ at point $x$. This sequence $\left\{x_{n}\right\}$ is also called the orbit of $f$ at point $x$. We will denote it by $O(x)$ and use $O(x, y)$ to denote set $O(x) \cup O(y)$.

Let $\Phi$ denote the set of all functions $\varphi:[0, \infty) \rightarrow[0, \infty)$ satisfying the following properties:

(a) $\varphi$ is monotone nondecreasing;

(b) $\lim \varphi^{n}(t)=0$ for any $t>0$.

The function $\varphi \in \Phi$ is known as the comparison function (see [29]). As a consequence of the above properties, we have the following (see [29]).

Lemma 7. If $\varphi \in \Phi$ then $\varphi(t)<t$ for all $t>0$ and $\varphi(0)=0$. 
Definition 8. If $(X, d)$ is a metric space and $f: X \rightarrow X$, then $f$ is called a

(1) contraction if there exists real number $\alpha \in(0,1)$ such that

$$
d(f(x), f(y)) \leqslant \alpha d(x, y) \quad \forall x, y \in X
$$

(2) $\varphi$-contraction if there exists a function $\varphi:[0, \infty) \rightarrow$ $[0, \infty)$ such that for any $x, y \in X$

$$
d(f(x), f(y)) \leqslant \varphi(d(x, y))
$$

(3) generalized $\varphi$-contraction if there exists a function $\varphi$ : $[0, \infty) \rightarrow[0, \infty)$ such that for any $x, y \in X$

$$
d(f(x), f(y)) \leqslant \varphi\left(d_{f}(x, y)\right),
$$

where

$$
d_{f}(x, y)=\max \{d(x, y), d(x, f(x)), d(y, f(y))\} .
$$

Lemma 9 (Aranđelović and Kečkić [2]). Let $X$ be a nonempty set, let $f: X \rightarrow X$, and let $n$ be a fixed positive integer such that the iterate $f^{n}$ has a unique fixed point $z$. Then

(1) $z$ is a unique fixed point of $f$;

(2) if $X$ is a topological space and any sequence of Picard iterates defined by $f^{n}$ converges to $z$, then the sequence of Picard iterates defined by $f$ always converges to $z$.

\section{Some Topological Results}

Proposition 10. Let $(X, d)$ be a symmetric space satisfying $(W)$. Then it satisfies (W4) and (JMS).

Proof. The implication (W) $\Rightarrow(\mathrm{W} 4)$ is straightforward (see [15]).

Now let $\left\{x_{n}\right\},\left\{y_{n}\right\}$ and $\left\{z_{n}\right\}$ be sequences in $X$ such that

$$
\begin{aligned}
& \lim _{n \rightarrow \infty} d\left(x_{n}, y_{n}\right)=0, \\
& \lim _{n \rightarrow \infty} d\left(y_{n}, z_{n}\right)=0 .
\end{aligned}
$$

From (W) it follows that

$$
\lim _{n \rightarrow \infty} d\left(x_{n}, z_{n}\right) \neq \infty
$$

So, $(X, d)$ satisfies (JMS).

A semi-metric space in which all balls $B(x, r), x \in X$ and $r>0$, are open will be called a semi-metric space with open balls.

Proposition 11. Let $(X, d)$ be a compact semi-metric space with open balls and $K \subseteq X$ a nonempty compact set. Then $K$ is bounded.
Proof. $\left(X, \tau_{d}\right)$ is first countable [2, Proposition 3] and $T_{1}$ space [2, page 5161]. Also, $(X, d)$ satisfies the property (SC) because all $B(x, r)$ are open sets [2, Theorem 1$]. K$ is countably compact because it is compact [30, Theorem 11.9]. It is sequentially compact, as a first countable countably compact set [30, Problem 10.7].

Suppose that $K$ is not bounded. Let $x_{0} \in K$. For each positive integer $n$ there exists $x_{n} \in K$ such that $d\left(x_{n}, x_{0}\right)>n$. Then there exists $x_{*} \in K$ and an increasing sequence of positive integers $n_{k}$ such that, in the topology $\tau_{d}$,

$$
\lim _{k \rightarrow \infty} x_{n_{k}}=x_{*} \text {, }
$$

because $K$ is sequentially compact. So, we get that

$$
\varlimsup_{k \rightarrow \infty} d\left(x_{n_{k}}, x_{0}\right) \leqslant d\left(x_{*}, x_{0}\right)
$$

which is a contradiction because

$$
\lim _{k \rightarrow \infty} d\left(x_{n_{k}}, x_{0}\right)=\infty
$$

\section{Bounded Semi-Metric Spaces: Fixed Point Results}

In this section, we obtain generalizations of fixed point results of Browder [19] and Walter [20] (see also [7]).

Theorem 12. Let $(X, d)$ be a bounded semi-metric and $d$ Cauchy complete space satisfying (W4). Suppose $f: X \rightarrow X$ satisfies that, for $x \in X$, there exists $\nu(x) \in \mathbb{N}$ such that, for any $v \geqslant v(x)$ and $y \in X$,

$$
d\left(f^{v}(x), f^{v}(y)\right) \leqslant \varphi(\operatorname{diam}(O(x, y)))
$$

with $\varphi \in \Phi$. Then there exists $z \in X$ such that $\lim _{p \rightarrow \infty} f^{p}(x)=$ $z$ in the topology $\tau_{d}$ (or equivalently $d\left(f^{p}(x), z\right) \rightarrow 0$ as $p \rightarrow$ $\infty), \forall x \in X$.

Proof. Let $\mu=\max \{\nu(x), \nu(y)\}$ and $\nu \geqslant \mu$.

Each element $\alpha \in O\left(f^{\mu}(x), f^{\mu}(y)\right)$ has one of forms $f^{\nu+\rho}(x)$ or $f^{\nu+\rho}(y)$, with $\rho \geqslant 0$. Now let $\alpha, \beta \in$ $O\left(f^{\mu}(x), f^{\mu}(y)\right)$ and consider the case with $\alpha=f^{\nu+\rho}(x)$ and $\beta=f^{\nu+\rho}(y)$; then

$$
\begin{aligned}
d(\alpha, \beta) & =d\left(f^{\nu+\rho}(x), f^{\nu+\rho}(y)\right) \\
& \leqslant \varphi\left(\operatorname{diam}\left(O\left(f^{\rho}(x), f^{\rho}(y)\right)\right)\right) \\
& \leqslant \varphi(\operatorname{diam}(O(x, y))) .
\end{aligned}
$$

Other cases will lead to the same inequality:

$$
\operatorname{diam}\left(O\left(f^{\mu}(x), f^{\mu}(y)\right)\right) \leqslant \varphi(\operatorname{diam}(O(x, y))) .
$$

Now, define the sequences $\left\{E_{n}\right\}_{n=0}^{\infty} \subset X$ and $\{p(n)\}_{n=0}^{\infty}$ by $p(0)=0, p(n+1)=p(n)+\max \left\{v\left(f^{p(n)}(x)\right), \nu\left(f^{p(n)}(y)\right)\right\}$, and $E_{n}=O\left(f^{p^{(n)}}(x), f^{p^{(n)}}(y)\right), n=0,1,2, \ldots$ 
We want to prove that

$$
\operatorname{diam}\left(E_{n+1}\right) \leqslant \varphi\left(\operatorname{diam}\left(E_{n}\right)\right), \quad n=0,1,2, \ldots
$$

By (20), we get that (21) is valid for $n=0$.

Now, let $n$ be arbitrary and set $\gamma=f^{p^{(n)}}(x), \xi=f^{p^{(n)}}(y)$, and $\eta=\max \left\{v\left(f^{p(n)}(x)\right), v\left(f^{p^{(n)}}(y)\right)\right\}$; then

$$
\begin{aligned}
& \operatorname{diam}\left(O\left(f^{\eta}(x), f^{\eta}(y)\right)\right) \\
& \quad \leqslant \varphi(\operatorname{diam}(O(\gamma, \xi))) \\
& \quad=\varphi\left(\operatorname{diam}\left(O\left(f^{p(n)}(x), f^{p(n)}(y)\right)\right)\right) \\
& \quad=\varphi\left(\operatorname{diam}\left(E_{n}\right)\right) .
\end{aligned}
$$

But $f^{\eta}(\gamma)=f^{p(n)+\eta}(x)=f^{p(n+1)}(x)$ and $f^{\eta}(\xi)=$ $f^{p(n)+\eta}(y)=f^{p(n+1)}(y)$. Thus $\operatorname{diam}\left(O\left(f^{\eta}(\gamma), f^{\eta}(\xi)\right)\right)=$ $\operatorname{diam}\left(O\left(f^{p(n+1)}(x), f^{p(n+1)}(y)\right)\right)=\operatorname{diam}\left(E_{n+1}\right)$.

Therefore (21) holds for all $n=0,1,2, \ldots$.

Now, by (21) and the monotonicity of $\varphi$, we get that $\operatorname{diam}\left(E_{n+1}\right) \leqslant \varphi^{(n+1)}\left(\operatorname{diam}\left(E_{0}\right)\right)=\varphi^{(n+1)}(\operatorname{diam}(O(x, y))) \rightarrow$ 0 as $n \rightarrow \infty$ that is, $\lim _{n \rightarrow \infty} \operatorname{diam}\left(E_{n}\right)=$ $\lim _{n \rightarrow \infty} \operatorname{diam}\left(O\left(f^{p(n)}(x), f^{p(n)}(y)\right)\right)=0$ which is equivalent to $\lim _{p \rightarrow \infty} \operatorname{diam}\left(O\left(f^{p}(x), f^{p}(y)\right)\right)=0$. But

$$
\begin{aligned}
& \lim _{p \rightarrow \infty} \operatorname{diam}\left(O\left(f^{p}(x)\right)\right) \\
& \quad \leqslant \lim _{p \rightarrow \infty} \operatorname{diam}\left(O\left(f^{p}(x), f^{p}(y)\right)\right)=0,
\end{aligned}
$$

which implies

$$
\lim _{p \rightarrow \infty} \operatorname{diam}\left(O\left(f^{p}(x)\right)\right)=0 .
$$

Similarly

$$
\lim _{p \rightarrow \infty} \operatorname{diam}\left(O\left(f^{p}(y)\right)\right)=0 .
$$

Hence, $\left\{f^{p}(x)\right\}$ and $\left\{f^{p}(y)\right\}$ are $d$-Cauchy sequences, and by the $d$-completeness of $X$, there exists $z, w \in X$ such that $\lim _{p \rightarrow \infty} f^{p}(x)=z$ and $\lim _{p \rightarrow \infty} f^{p}(y)=w$ in the topology $\tau_{d}$.

Since $\lim _{p \rightarrow \infty} d\left(f^{p}(x), z\right)=0$ and $\lim _{p \rightarrow \infty} d\left(f^{p}(x)\right.$, $\left.f^{p}(y)\right)=0$, (W4) implies that $\lim _{p \rightarrow \infty} d\left(f^{p}(y), z\right)=$ 0 . But $\lim _{p \rightarrow \infty} f^{\mathrm{P}}(y)=w$ in the topology $\tau_{d}$ and so $\lim _{p \rightarrow \infty} d\left(f^{p}(y), w\right)=0$. Since (W4) implies (W3), we have $z=w$. Since $y$ is arbitrary in $X, \lim _{p \rightarrow \infty} f^{p}(x)=z$ in the topology $\tau_{d}, \forall x \in X$.

Corollary 13. If, in addition to the hypothesis of Theorem 12, one assumes that $f$ is $\tau_{d}$-continuous (i.e, $x_{n} \rightarrow x$ in the topology $\tau_{d}$ implies $f\left(x_{n}\right) \rightarrow f(x)$ in the topology $\left.\tau_{d}\right)$ then $f$ has a fixed point.

Proof. Since $\lim _{p \rightarrow \infty} f^{p}(x)=z$ in the topology $\tau_{d}$, by the $\tau_{d^{-}}$ continuity of $f, \lim _{p \rightarrow \infty} f^{p+1}(x)=f(z)$ in the topology $\tau_{d}$. Therefore, since (W4) implies (W3), $f(z)=z$. Hence, $z \in X$ is a fixed point.
Theorem 14. Let $(X, d)$ be a bounded semi-metric and $d$ Cauchy complete space satisfying (W4), (CC) and (JMS). Suppose that $f$ is a self-map on $X$, and for $x, y \in X$.

$$
d(f(x), f(y)) \leqslant \varphi(\operatorname{diam}(O(x, y))) .
$$

Then $f$ has a unique fixed point and $\lim _{p \rightarrow \infty} f^{p}(x)=z$ in the topology $\tau_{d}$ (or equivalently $d\left(f^{p}(x), z\right) \rightarrow 0$ as $p \rightarrow \infty$ ), $\forall x \in X$.

Proof. By Theorem 12, there exists $z \in X$ such that $\lim _{p \rightarrow \infty} d\left(f^{p}(x), z\right)=0$ for all $x \in X$.

Next, assume that $z \neq f(z)$; that is, $\operatorname{diam}(O(z))=\beta>0$.

Thus, it is possible to choose two sequences $\{i(p)\},\{j(p)\}$ such that

$$
\lim _{p \rightarrow \infty} d\left(f^{i(p)}(z), f^{j(p)}(z)\right)=\beta
$$

So one can pick $\delta>0$ with a corresponding $\eta>0$, such that $\eta \leqslant \beta / 2$.

Since there exists $p_{0} \in \mathbb{N}$ such that

$$
d\left(f^{n}(z), z\right) \leqslant \frac{\delta}{2}, \quad d\left(f^{m}(z),(z)\right) \leqslant \frac{\delta}{2}, \quad \forall n, m \geqslant p_{0},
$$

therefore $d\left(f^{n}(z), z\right)+d\left(f^{m}(z), z\right) \leqslant \delta$, by Proposition 1(ii)

$$
d\left(f^{n}(z), f^{m}(z)\right) \leqslant \eta \leqslant \frac{\beta}{2}, \quad \forall n, m \geqslant p_{0} .
$$

So, $i(p) \equiv i$ for infinitely many $p$, with $0 \leqslant i \leqslant$ $p_{0}$; thus there exists a sequence $\{r(p)\} \subseteq\{j(p)\}$ such that $\lim _{p \rightarrow \infty} d\left(f^{i}(z), f^{r(p)}(z)\right)=\beta$. So, either $r(p) \equiv j$ for infinitely many $p$ (i.e., $d\left(f^{i}(z), f^{j}(z)\right)=\beta$ ) or there exists a sequence $\{s(p)\} \subseteq\{r(p)\}$ with $s(p) \rightarrow \infty$ as $p \rightarrow \infty$ which implies $d\left(f^{i}(z), z\right)=\beta$.

In both cases, one can conclude that there exists $i, j \geqslant 0$ such that $d\left(f^{i}(z), f^{j}(z)\right)=\beta$.

If $d\left(z, f^{j}(z)\right)=\beta$, since $\lim _{p \rightarrow \infty} d\left(f^{p}(x), z\right)=0$ and by (CC) of $d$, we get

$$
\begin{aligned}
\beta & =d\left(z, f^{j}(z)\right) \\
& =\lim _{p \rightarrow \infty} d\left(f^{p}(z), f^{j}(z)\right) \\
& \leqslant \lim _{p \rightarrow \infty} \varphi\left(\operatorname{diam}\left(O\left(f^{p-1}(z), f^{j-1}(z)\right)\right)\right) \\
& \leqslant \lim _{p \rightarrow \infty} \varphi(\operatorname{diam}(O(z)))=\varphi(\beta),
\end{aligned}
$$

which is a contradiction with $\varphi(\beta)<\beta$, for $\beta>0$.

On the other hand, if $i, j \geqslant 1$, by (26),

$$
\begin{aligned}
\beta & =d\left(f^{i}(z), f^{j}(z)\right) \\
& \leqslant \varphi\left(\operatorname{diam}\left(O\left(f^{i-1}(z), f^{j-1}(z)\right)\right)\right) \\
& \leqslant \varphi(\beta)
\end{aligned}
$$

which is also a contradiction. Hence $\beta=0$; that is, $f(z)=$ $z$. 
Corollary 15. Let $(X, d)$ be a bounded semi-metric and $d$ Cauchy complete space satisfying $(W)$ and (CC). Suppose that $f$ is a self-map on $X$, and for $x, y \in X$

$$
d(f(x), f(y)) \leqslant \varphi(\operatorname{diam}(O(x, y))) .
$$

Then $f$ has a unique fixed point and $\lim _{p \rightarrow \infty} f^{p}(x)=z$ in the topology $\tau_{d}$ (or equivalentlyd $\left(f^{p}(x), z\right) \rightarrow 0$ as $p \rightarrow \infty$ ), for all $x \in X$.

\section{Symmetric Spaces: Fixed Point Results}

In this section, we extend results attributed to Maiti et al. [21, Theorem 4] and Aranđelović and Kečkić [2, Theorem 3].

Theorem 16. Let $(X, d)$ be a d-Cauchy complete symmetric space satisfying (W3) and (JMS). Let $f: X \rightarrow X$ be a $\tau_{d^{-}}$ continuous map such that

$$
d(f(x), f(y)) \leqslant \varphi\left(d_{f}(x, y)\right),
$$

for all $x, y \in X$, and $\varphi \in \Phi$. Then $f$ has a unique fixed point $z \in X$ and for each $x \in X$, the sequence of Picard iterates defined by $f$ at $x$ converges to $z$ in the topology $\tau_{d}$.

Proof. Define $d^{*}: X \times X$ as follows: $d^{*}(x, y)=0$ for $x=y$ and $d^{*}(x, y)=d_{f}(x, y)$ otherwise. Then the space $\left(X, d^{*}\right)$ is a symmetric space. Also, we have $d(x, y) \leqslant d^{*}(x, y)$ for any $x, y \in X$. So, if $\left\{x_{n}\right\} \subseteq X$ is an arbitrary $d^{*}$-Cauchy sequence in $\left(X, d^{*}\right)$, then $\left\{x_{n}\right\}$ is a $d$-Cauchy sequence in $(X, d)$.

Let $x, y \in X$. From

$$
\begin{aligned}
& d\left(f^{2}(x), f(x)\right) \leqslant \varphi(d(x, f(x))), \\
& d\left(f^{2}(y), f(y)\right) \leqslant \varphi(d(y, f(y))), \\
& d(f(x), f(y)) \leqslant \varphi\left(d_{f}(x, y)\right),
\end{aligned}
$$

it follows that

$$
d^{*}(f(x), f(y)) \leqslant \varphi\left(d^{*}(x, y)\right) .
$$

So $f$ is a $\varphi$-contraction on $\left(X, d^{*}\right)$.

Let $\delta, \eta$ be defined as in (ii) of Proposition 1. Then there exists the least positive integer $j \geqslant 1$ such that $\varphi^{j}(\eta) \leqslant \delta / 2$.

Let $g=f^{j}$. We have that $g$ is continuous (in $\tau_{d}$ ). Then

$$
\begin{aligned}
d^{*} & (g(x), g(y)) \\
& =d^{*}\left(f\left(f^{j-1}(x)\right), f\left(f^{j-1}(y)\right)\right) \\
& \leq \varphi\left(d^{*}\left(f^{j-1}(x), f^{j-1}(y)\right)\right) \\
& \leqslant \varphi^{j}\left(d^{*}(x, y)\right) .
\end{aligned}
$$

So $g$ is a $\varphi^{j}$-contraction on $\left(X, d^{*}\right)$.

Let $x \in X$ and $\psi=\varphi^{j}$. Then $\psi \in \Phi$ and

$$
\begin{array}{r}
d^{*}\left(g^{m+n}(x), g^{n}(x)\right) \leqslant \psi^{n}\left(d^{*}\left(x, g^{m}(x)\right)\right) \\
\text { for any } m, n \in \mathbb{N} .
\end{array}
$$

So

$$
d^{*}\left(g^{n+1}(x), g^{n}(x)\right) \leqslant \psi^{n}\left(d^{*}(x, g(x))\right)
$$

which implies that

$$
d^{*}\left(g^{n+1}(x), g^{n}(x)\right) \longrightarrow 0 .
$$

Then there exists $k \in \mathbb{N}$ such that

$$
d^{*}\left(g^{k}(x), g^{k+1}(x)\right) \leqslant \min \left\{\frac{\delta}{2}, \eta\right\} .
$$

We will prove that, for all $n \in \mathbb{N}$,

$$
d^{*}\left(g^{k}(x), g^{k+n}(x)\right) \leqslant \eta .
$$

By definition of $k$, we get that (41) is valid for $n=1$. Now, assume that (41) is satisfied for some $n \in \mathbb{N}$. From

$$
\begin{aligned}
& d^{*}\left(g^{k}(x), g^{k+1}(x)\right) \leqslant \frac{\delta}{2}, \\
& d^{*}\left(g^{k+1}(x), g^{k+n+1}(x)\right) \\
& \quad \leqslant \psi\left(d^{*}\left(g^{k}(x), g^{k+n}(x)\right)\right) \\
& \quad \leqslant \psi(\eta) \leqslant \frac{\delta}{2},
\end{aligned}
$$

it follows that

$$
d^{*}\left(g^{k}(x), g^{k+1}(x)\right)+d^{*}\left(g^{k+1}(x), g^{k+n+1}(x)\right) \leqslant \delta,
$$

which by Proposition 1 implies that

$$
d^{*}\left(g^{k}(x), g^{k+n+1}(x)\right) \leqslant \eta .
$$

So, by induction we get that (41) is satisfied for any $n \geqslant 1$. Thus

$$
d^{*}\left(g^{k+n}(x), g^{k+n+m}(x)\right) \leqslant \psi^{n}(\eta), \quad \text { for any } m, n \in \mathbb{N} .
$$

Hence $\left\{g^{n}(x)\right\}$ is a $d^{*}$-Cauchy sequence in $\left(X, d^{*}\right)$, which implies that $\left\{g^{n}(x)\right\}$ is a $d$-Cauchy sequence in $(X, d)$. It follows that there exists $z \in X$ such that $\lim _{n \rightarrow \infty} g^{n}(x)=z$ (in the topology $\tau_{d}$ ) because $(X, d)$ is $d$-Cauchy complete. Then $\lim _{n \rightarrow \infty} g^{n+1}(x)=g(z)$ (in the topology $\tau_{d}$ ) because $g$ is $\tau_{d^{-}}$ continuous. Now we get that $g(z)=z$ because $(X, d)$ satisfies (W3).

If $y$ is another fixed point of $f$, then for all $n$ we have

$$
\begin{array}{r}
d^{*}(y, z)=d^{*}\left(g^{n}(y), g^{n}(z)\right) \leqslant \psi^{n}\left(d^{*}(y, z)\right) \longrightarrow 0, \\
\text { as } n \longrightarrow \infty .
\end{array}
$$

So $z$ is a unique fixed point of $g$. By Lemma 9 we get that $z$ is a unique fixed point of $f$.

From

$$
\begin{array}{r}
d^{*}\left(z, g^{n+1}(x)\right) \leqslant \varphi\left(\operatorname { m a x } \left\{d(z, g(z)), d\left(z, g^{n}(x)\right),\right.\right. \\
\left.\left.d\left(g^{n}(x), g^{n+1}(x)\right)\right\}\right)
\end{array}
$$

it follows that for each $x \in X$ the sequence of Picard iterates defined by $g=f^{j}$ at $x$ converges, in the topology $\tau_{d^{*}}$, to $z$, which implies their convergence in the topology $\tau_{d}$. So, by Lemma 9, we obtain that for each $x \in X$ the sequence of Picard iterates defined by $f$ at $x$ converges, in the topology $\tau_{d}$, to $z$. 
Remark 17. The next example of [10] illustrates that the continuity of $f$ in Theorem 16 can not be omitted.

Example 18. Let $X=\{0\} \cup\{1 / n: n \in \mathbb{N}\}$ and let $d$ be defined as follows:

$$
\begin{aligned}
& d(0,1)=d(1,0)=1 ; \\
& d(1,1 / n)=d(1 / n, 1)=2 / 3 \text { for } n \geqslant 2 ; \\
& d(1,1)=0 ; \text { otherwise } d(x, y)=|x-y| .
\end{aligned}
$$

Let $f: X \rightarrow X$ given by

$$
f(x)= \begin{cases}\frac{x}{4}, & \text { for } x \neq 0 \\ 1, & \text { for } x=0\end{cases}
$$

Then $(X, d)$ is a bounded $d$-Cauchy complete semi-metric space and

$$
d(f(x), f(y)) \leqslant \varphi\left(d_{f}(x, y)\right)
$$

for all $x, y \in X$, (see [10, Example 3]). (X,d) satisfies (W3) and (JMS).

But $f$ does not have a fixed point in $X$. Note that $f$ is not continuous.

\section{Applications}

We now present applications of our results to probabilistic spaces. We begin with some essential definitions.

Definition 19. Let $X$ be a set and $\mathscr{F}$ a mapping of $X \times X$ into a collection $\mathscr{L}$ of all distribution functions $F$ (a distribution function $F$ is a nondecreasing and left continuous mapping of reals into $[0,1]$ with $\inf \{F(x)\}=0$ and $\sup \{F(x)\}=1$ ). Consider the following conditions:

(I) $F_{x, y}(0)=0$ for all $x, y \in X$, where $F_{x, y}$ denotes the value of $\mathscr{F}$ at $(x, y) \in X \times X$.

(II) $F_{x, y}=H$ if and only if $x=y$, where $H$ denotes the distribution function defined by $H(x)=0$ if $x \leqslant 0$ and $H(x)=1$ if $x>0$.

(III) $F_{x, y}=F_{y, x}$.

(IV) If $F_{x, y}(\varepsilon)=1$ and $F_{y, z}(\delta)=1$, then $F_{x, z}(\varepsilon+\delta)=1$.

If $\mathscr{F}$ satisfies (I) and (II), then it is called a PPMstructure on $X$ and the pair $(X, \mathfrak{F})$ is called a PPM-space. $\mathfrak{F}$ satisfying (III) is said to be symmetric. A symmetric PPMspace satisfying (IV) is a probabilistic metric space (or briefly PM-space).

The topology $\tau_{\mathfrak{F}}$ in $(X, \mathfrak{F})$ is generated by the family

$$
\mathcal{U}=\left\{U_{x}(\varepsilon, \lambda): x \in X, \varepsilon>0, \lambda>0\right\},
$$

where the set

$$
U_{x}(\varepsilon, \lambda)=\left\{y \in X: F_{x, y}(\varepsilon)>1-\lambda\right\}
$$

is called $(\varepsilon, \lambda)$-neighborhood of $x \in X$. A sequence $\left\{x_{n}\right\}$ is said to be a Cauchy sequence if, for every given $\varepsilon, \lambda>0$, there exists a positive integer $n_{0}=n_{0}(\varepsilon, \lambda)$ such that $F_{x_{n}, x_{m}}(\varepsilon)>$ $1-\lambda$ for all $m, n \geqslant n_{0}$. A $T_{1}$ topology $\tau_{\mathfrak{F}}$ on $X$ is defined as follows: $U \in \tau_{\widetilde{F}}$ if, for any $x \in U$, there exists $\varepsilon>0$ such that $U_{x}(\varepsilon, \varepsilon) \subset U$. If $U_{x}(\varepsilon, \varepsilon) \in \tau_{\mathfrak{F}}$, then $\tau_{\mathfrak{F}}$ is said to be topological.

The space $(X, \mathfrak{F})$ is called $\mathfrak{F}$-complete if for every Cauchy sequence $\left\{x_{n}\right\}$ there exists $x \in X$ such that $\lim _{n \rightarrow \infty} F_{x_{n}, x}(\varepsilon)=$ 1 for all $\varepsilon>0$.

Remark 20. (1) The condition (W) is equivalent to

$$
\begin{array}{r}
\lim _{n \rightarrow \infty} F_{x_{n}, y_{n}}(\varepsilon)=1, \quad \lim _{n \rightarrow \infty} F_{y_{n}, z_{n}}(\varepsilon)=1, \\
\text { imply } \lim _{n \rightarrow \infty} F_{x_{n}, z_{n}}(\varepsilon)=1 .
\end{array}
$$

(2) The condition (W4) is equivalent to

$$
\begin{array}{r}
\lim _{n \rightarrow \infty} F_{x_{n}, x}(\varepsilon)=1, \quad \lim _{n \rightarrow \infty} F_{x_{n}, y_{n}}(\varepsilon)=1, \\
\text { imply } \lim _{n \rightarrow \infty} F_{y_{n}, x}(\varepsilon)=1 .
\end{array}
$$

The following lemma was proved in [11].

Lemma 21 (Hicks and Rhoades [11]). Let $(X, \mathfrak{F})$ be a symmetric PPM-space. Set

$$
\begin{aligned}
& d(x, y) \\
& \quad \begin{cases}0, & \text { if } y \notin U_{x}(\varepsilon, \varepsilon), \forall \varepsilon>0 \\
\sup \left\{\varepsilon: y \notin U_{x}(\varepsilon, \varepsilon),\right. & \text { otherwise. } \\
\varepsilon>0\}, & \end{cases}
\end{aligned}
$$

Then $d$ is a bounded compatible symmetric for $X$.

Lemma 22 (Hicks and Rhoades [11]). Let $(X, \mathfrak{F})$ be a symmetric PPM-space. Defined as in (52). Then

(1) $d(x, y)<t$ if and only if $F_{x, y}(t)>1-t$;

(2) $d$ is compatible symmetric for $\tau_{\mathfrak{F}}$;

(3) $(X, \mathfrak{F})$ is complete if and only if $(X, d)$ is d-Cauchy complete symmetric space;

(4) if $\tau_{\widetilde{\mho}}$ is topological, $d$ is semi-metric.

$f:(X, \mathscr{F}) \rightarrow(X, \mathscr{F})$ is $\mathscr{F}$-continuous if $F_{x_{n}, x}(t) \rightarrow 1$ for all $t>0$ implies $F_{f\left(x_{n}\right), f(x)}(t) \rightarrow 1$. This is equivalent to the continuity of $f:(X, d) \rightarrow(X, d)$, where $d$ is as in Lemma 21.

Let $\Phi^{\prime}$ denote the set of all functions $\varphi \in \Phi$ satisfying

$$
\lim _{\epsilon \rightarrow 0} \varphi(t+\epsilon)=\varphi(t)
$$

for all $t>0$.

Theorem 23. Let $(X, \mathfrak{F})$ be a complete symmetric PPM-space that satisfies $(P 4)$, where $\tau_{\mathfrak{r}}$ is a topological. Suppose $f: X \rightarrow$ 
$X$ is $\mathscr{F}$-continuous and satisfies that for $x \in X$ there exists $\nu(x) \in \mathbb{N}$ such that for any $v \geqslant v(x)$ and $y \in X$

$$
\begin{array}{r}
F_{u, v}(t)>1-t \quad \text { implies } F_{f^{v}(x), f^{v}(y)}(\varphi(t))>1-\varphi(t), \\
u, v \in O(x, y)
\end{array}
$$

for every $t>0$ with $\varphi \in \Phi^{\prime}$. Then $f$ has a fixed point.

Proof. Define $d$ as in (52). According to Lemmas 21 and 22, $(X, d)$ is a bounded $d$-Cauchy complete semi-metric space satisfying (W4). Now assume that (54) is satisfied. Let $\varepsilon>0$ be given and let $t=d(u, v)+\varepsilon$. Then $d(u, v)<t$ gives $F_{u, v}(t)>1-t$ and so $F_{f^{v}(x), f^{v}(y)}(\varphi(t))>1-\varphi(t)$ and so $d\left(f^{v}(x), f^{v}(y)\right)<\varphi(t)=\varphi(d(u, v)+\varepsilon)$. Since $\varepsilon$ was arbitrary, we have that

$$
\begin{aligned}
d\left(f^{v}(x), f^{v}(y)\right) & \leqslant \varphi(d(u, v)) \\
& \leqslant \varphi\left(\sup _{u, v \in O(x, y)} d(u, v)\right) \\
& =\varphi(\operatorname{diam}(O(x, y))) .
\end{aligned}
$$

By Corollary 13, $f$ has a fixed point.

Theorem 24. Let $(X, \mathfrak{F})$ be a complete symmetric PPM-space that satisfies $(P)$. Let $f: X \rightarrow X$ be $\mathscr{F}$-continuous such that

$$
F_{x, y}(t)>1-t \quad \text { implies } F_{f(x), f(y)}(\varphi(t))>1-\varphi(t)
$$

for all $x, y \in X$, and $\varphi \in \Phi^{\prime}$. Then $f$ has a unique fixed point.

Proof. Define $d$ as in (52). According to Lemma 21, $d$ is a bounded compatible symmetric for $\tau_{\widetilde{F}}$ and $(X, d)$ is $d$-Cauchy complete symmetric space satisfying (W3) and (JMS). Suppose (56) is satisfied and let $\varepsilon>0$ be given. Let $t=$ $\max \{d(x, y)+\varepsilon, d(x, f(x)), d(y, f(y))\}$. Then $d(x, y)+\varepsilon \leqslant$ $t$ and so $d(x, y) \leqslant t-\varepsilon<t$, which implies $F_{x, y}(t)>$ $1-t$. This further implies that $F_{f(x), f(y)}(\varphi(t))>1-\varphi(t)$ and so

$$
\begin{gathered}
d(f(x), f(y)) \\
<\varphi(t)=\varphi(\max \{d(x, y)+\varepsilon, d(x, f(x)), d(y, f(y))\}) \\
=\max \{\varphi(d(x, y)+\varepsilon), \varphi(d(x, f(x))), \\
\varphi(d(y, f(y)))\} .
\end{gathered}
$$

Since $\varepsilon$ was arbitrary, we have that

$$
\begin{aligned}
d( & f(x), f(y)) \\
& \leqslant \max \{\varphi(d(x, y)), \varphi(d(x, f(x))), \varphi(d(y, f(y)))\} \\
& =\varphi(\max \{d(x, y), d(x, f(x)), d(y, f(y))\}) .
\end{aligned}
$$

Now Theorem 16 guarantees that $f$ has a unique fixed point $z \in X$.

\section{Some Open Problems}

Problem 25 (see [2]). Let $(X, d)$ be a symmetric space which satisfies the property (MT). Is it a semi-metric space (not necessarily with open balls)?

Problem 26. Does Theorem 16 hold if $d_{f}(x, y)$ is replaced with

$$
\begin{gathered}
D_{f}(x, y)=\max \{d(x, y), d(x, f(x)), d(y, f(y)), \\
d(x, f(y)), d(f(x), y)\} ?
\end{gathered}
$$

\section{Conflict of Interests}

The authors declare that there is no conflict of interests regarding the publication of this paper.

\section{Acknowledgments}

The authors would like to thank the reviewers for their valuable suggestions. This paper was funded by the Deanship of Scientific Research (DSR), King Abdulaziz University, Jeddah. The first and third authors acknowledge with thanks DSR for the financial support. The second author was supported by the Ministry of Education, Science and Technological Development of Serbia, Grant no. 174002.

\section{References}

[1] W. A. Wilson, "On semi-metric spaces," American Journal of Mathematics, vol. 53, no. 2, pp. 361-373, 1931.

[2] I. D. Aranđelović and D. J. Kečkić, "Symmetric spaces approach to some fixed point results," Nonlinear Analysis: Theory, Methods \& Applications, vol. 75, no. 13, pp. 5157-5168, 2012.

[3] M. A. Alghamdi, N. Shahzad, and O. Valero, "On fixed point theory in partial metric spaces," Fixed Point Theory and Applications, vol. 2012, article 175, 25 pages, 2012.

[4] R. P. Agarwal, M. A. Alghamdi, and N. Shahzad, "Fixed point theory for cyclic generalized contractions in partial metric spaces," Fixed Point Theory and Applications, vol. 2012, article 40, 11 pages, 2012.

[5] R. P. Agarwal, D. O’Regan, and N. Shahzad, "Fixed point theory for generalized contractive maps of Meir-Keeler type," Mathematische Nachrichten, vol. 276, pp. 3-22, 2004.

[6] Z. Kadelburg, S. Radenovic, and N. Shahzad, "A note on various classes of compatible-type pairs of mappings and common fixed point theorems," Abstract and Applied Analysis, vol. 2013, Article ID 697151, 6 pages, 2013.

[7] W. A. Kirk, "Contraction mappings and extensions," in Handbook of Metric Fixed Point Theory, pp. 1-34, Kluwer Academic Publishers, Dordrecht, The Netherlands, 2001.

[8] W. A. Kirk and N. Shahzad, "Generalized metrics and Caristi's theorem," Fixed Point Theory and Applications, vol. 2013, article 129, 9 pages, 2013.

[9] Sh. Rezapour, R. H. Haghi, and N. Shahzad, "Some notes on fixed points of quasi-contraction maps," Applied Mathematics Letters, vol. 23, no. 4, pp. 498-502, 2010. 
[10] J. Jachymski, J. Matkowski, and T. Świątkowski, "Nonlinear contractions on semimetric spaces," Journal of Applied Analysis, vol. 1, no. 2, pp. 125-134, 1995.

[11] T. L. Hicks and B. E. Rhoades, "Fixed point theory in symmetric spaces with applications to probabilistic spaces," Nonlinear Analysis: Theory, Methods \& Applications, vol. 36, no. 3, pp. 331344, 1999.

[12] M. Aamri and D. El Moutawakil, "Common fixed points under contractive conditions in symmetric spaces," Applied Mathematics E-Notes, vol. 3, pp. 156-162, 2003.

[13] M. Aamri, A. Bassou, and D. EL Moutawakil, "Common fixed points for weakly compatible maps in symmetric spaces with application to probabilistic spaces," Applied Mathematics ENotes, vol. 5, pp. 171-175, 2005.

[14] J. Zhu, Y. J. Cho, and S. M. Kang, "Equivalent contractive conditions in symmetric spaces," Computers \& Mathematics with Applications, vol. 50, no. 10-12, pp. 1621-1628, 2005.

[15] D. Miheț, "A note on a paper of Hicks and Rhoades," Nonlinear Analysis: Theory, Methods \& Applications, vol. 65, no. 7, pp. 14111413, 2006.

[16] M. Imdad, J. Ali, and L. Khan, "Coincidence and fixed points in symmetric spaces under strict contractions," Journal of Mathematical Analysis and Applications, vol. 320, no. 1, pp. 352360, 2006, Erratum in Journal of Mathematical Analysis and Applications, vol. 329, no. 1, p. 752, 2007.

[17] A. Aliouche, "A common fixed point theorem for weakly compatible mappings in symmetric spaces satisfying a contractive condition of integral type," Journal of Mathematical Analysis and Applications, vol. 322, no. 2, pp. 796-802, 2006.

[18] S. Radenović and Z. Kadelburg, "Quasi-contractions on symmetric and cone symmetric spaces," Banach Journal of Mathematical Analysis, vol. 5, no. 1, pp. 38-50, 2011.

[19] F. E. Browder, "Remarks on fixed point theorems of contractive type," Nonlinear Analysis, vol. 3, no. 5, pp. 657-661, 1979.

[20] W. Walter, "Remarks on a paper by F. Browder about contraction," Nonlinear Analysis: Theory, Methods \& Applications, vol. 5, no. 1, pp. 21-25, 1981.

[21] M. Maiti, J. Achari, and T. K. Pal, "Remarks on some fixed point theorems," Publications de l'Institut Mathématique, vol. 21, pp. 115-118, 1977.

[22] S. H. Cho, G. Y. Lee, and J. S. Bae, "On coincidence and fixedpoint theorems in symmetric spaces," Fixed Point Theory and Applications, vol. 2008, Article ID 562130, 9 pages, 2008.

[23] C. J. R. Borges, "On continuously semimetrizable and stratifiable spaces," Proceedings of the American Mathematical Society, vol. 24, pp. 193-196, 1970.

[24] S. Czerwik, "Contraction mappings in b-metric spaces," Acta Mathematica et Informatica Universitatis Ostraviensis, vol. 1, pp. 5-11, 1993.

[25] M. Jovanović, Z. Kadelburg, and S. Radenović, "Common fixed point results in metric-type spaces," Fixed Point Theory and Applications, vol. 2010, Article ID 978121, 15 pages, 2010.

[26] R. W. Heath, "A regular semi-metric space for which there is no semi-metric under which all spheres are open," Proceedings of the American Mathematical Society, vol. 12, pp. 810-811, 1961.

[27] F. Galvin and S. D. Shore, "Completeness in semimetric spaces," Pacific Journal of Mathematics, vol. 113, no. 1, pp. 67-75, 1984.

[28] L. F. McAuley, "A relation between perfect separability, completeness, and normality in semi-metric spaces," Pacific Journal of Mathematics, vol. 6, pp. 315-326, 1956.
[29] I. A. Rus, A. Petrusel, and G. Petrusel, Fixed Point Theory, Cluj University Press, Cluj-Napoca, Romania, 2008.

[30] S. Lipschutz, General Topology, McGraw-Hill, New York, NY, USA, 1965. 




Advances in

Operations Research

mansans

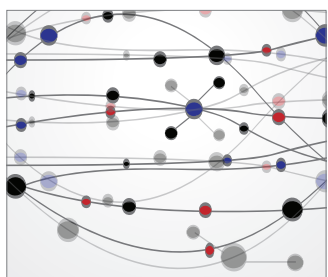

The Scientific World Journal
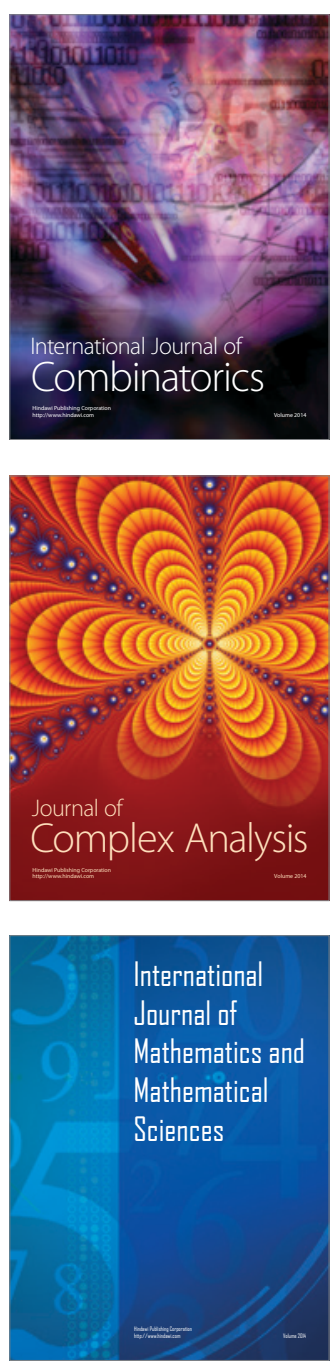
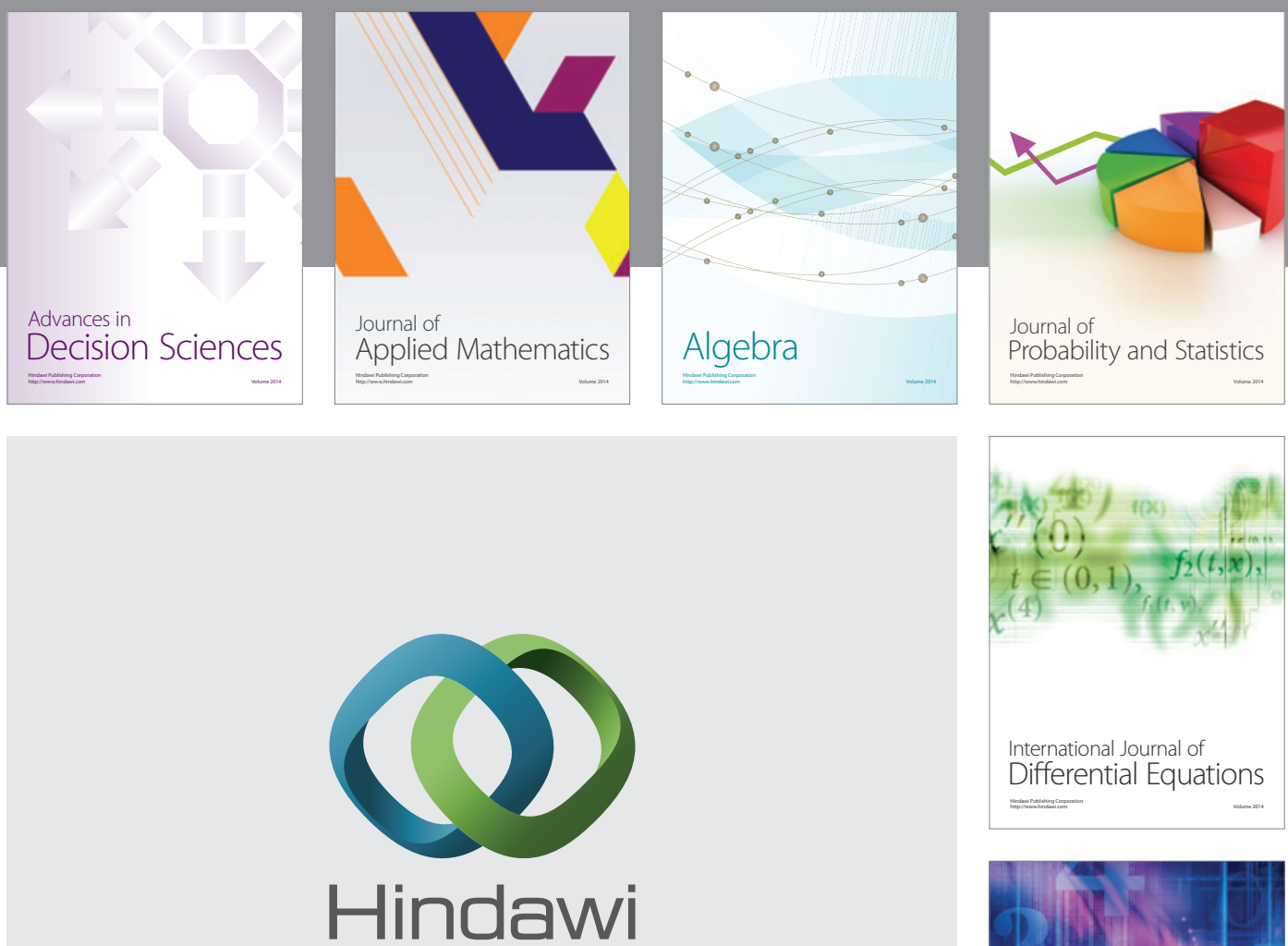

Submit your manuscripts at http://www.hindawi.com
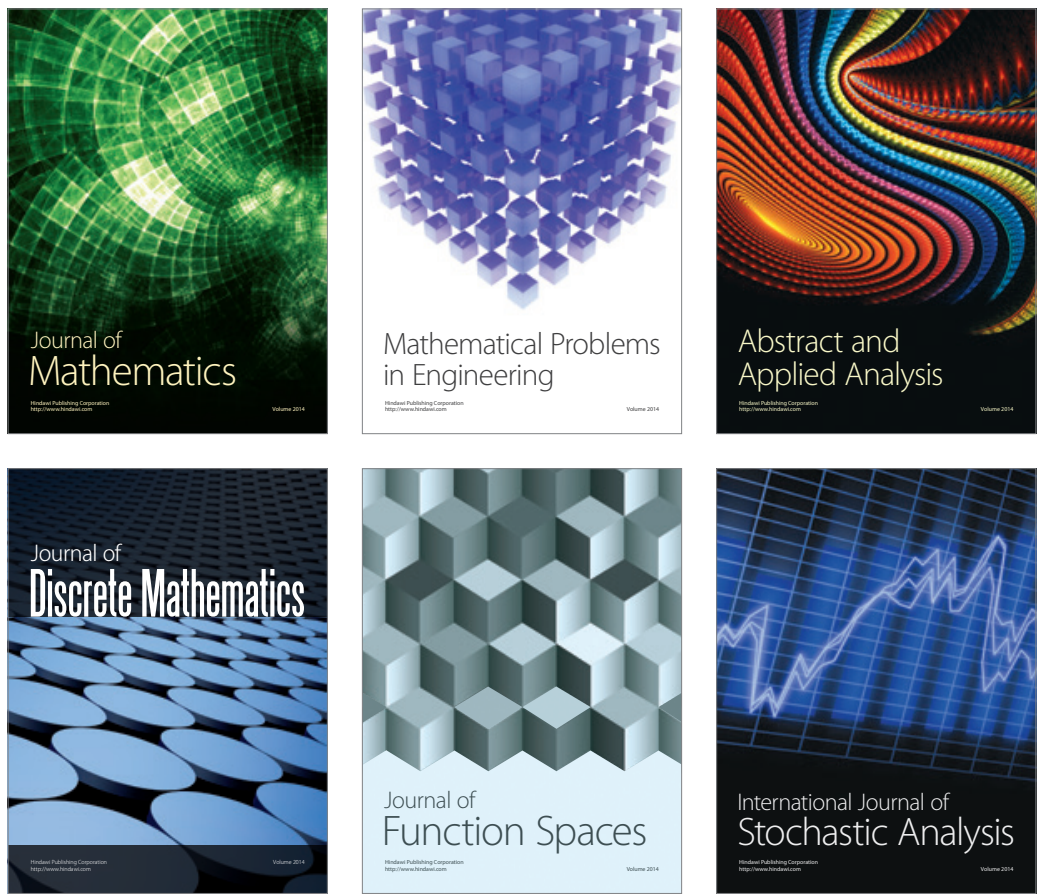

Journal of

Function Spaces

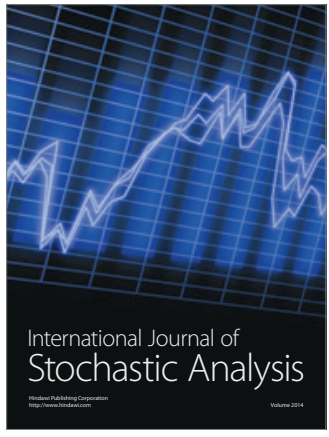


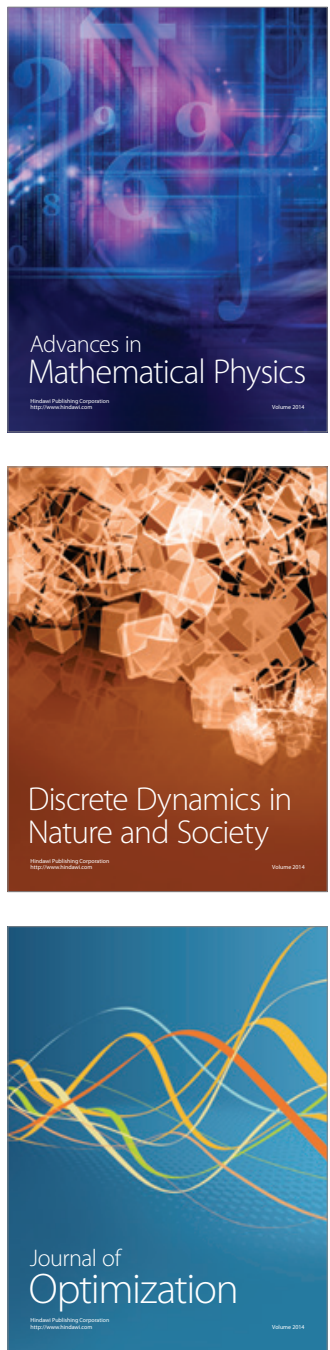